\title{
Conferència \\ Storie connesse nel Mediterraneo dell'età moderna: Asia, Africa e America
}

\author{
LuCA Lo BAsso \\ Università degli Studi di Genova
}

\section{Introduzione}

È possibile proporre oggi nuove interpretazioni storiografiche sul Mediterraneo in età moderna senza essere schiacciati, annullati, dal capolavoro di Fernand Braudel? Senza dubbio le pagine di Civiltà e Imperi del Mediterraneo all'epoca di Filippo II rimangono, nonostante siano passati settant'anni dalla prima edizione, di grande attualità, per come riescono ad ispirare ancora agli storici nuove chiavi di lettura, come quelle, oggi, che fanno riferimento alla storia marittima e alla storia globale. Il Mediterraneo braudeliano non solo suggerì alla storiografia mondiale l'approccio marittimistico, che ancora oggi fa fatica ad imporsi, ma spinse già all'epoca gli studiosi a ragionare sempre più in senso globale. Il Mediterraneo allargato divenne pertanto la chiave di lettura per un mondo, quello del Novecento, sempre più globalizzato e sempre più connesso. ${ }^{\mathrm{I}}$

Il Mediterraneo, crocevia naturale tra tre mondi - come scrisse Paul Valery - era un naturale connettore tra le diverse civiltà, tra le differenti

I. Giuliana Gemelli, Fernand Braudel e l'Europa universale, Marsilio, Venezia, 1990. 
economie, tra le molteplici culture e religioni. Un continente liquido, il Mediterraneo, unico per posizione e per storia, fatto di "guerre, commerce, échanges, volontaires ou non, de choses, de connaissances, de méthodes; mélanges de sang, de vocables, de légendes ou de traditions».2

Negli ultimi tempi, per meglio concettualizzare in senso storiografico queste suggestioni poetiche, gli storici hanno cominciato ad usare il termine "connessione» per categorizzare meglio i contatti, i commerci, le unioni, ma anche i contrasti, tra le diverse civiltà e le differenti culture del mondo mediterraneo. Il Mare Nostrum si è, piano piano, trasformato da un mero luogo geografico ad un vero e proprio paradigma interpretativo della storia, oggi inevitabilmente globale.

In primo battuta, alla fine degli anni Novanta del Novecento, fu lo storico indiano Sanjay Subrahmanyam a proporre il concetto di storie connesse, definizione poi ripresa e sviluppata da Serge Gruzinski, con la quale si intendeva in senso lato il collegamento tra i fenomeni del passato che in molti casi la storiografia studiava separatamente in maniera artificiale. Storie, dunque, tra diverse culture del mondo, che per converso andavano lette in maniera globale. ${ }^{3}$ Il paradigma iniziale di una nuova storia destinata a far superare il modello interpretativo tradizionale di tipo eurocentrico.

La connettività di Subrahmanyam prese con il tempo altri molteplici significati. Nella fattispecie, nella nota opera di Horden e Purcell del 2000, le connessioni si trasformarono nei legami commerciali (le rotte), culturali e religiosi tra le città portuali del Mediterraneo in epo-

2. Paul Valery, Euvres, I, Gallimard, Paris, 1957, p. I096; J. Carpentier e F. Lebrun, eds., Histoire de la Méditerranée, Seuil, Paris, 200I, p. I2; Fernand Braudel, Il Mediterraneo. Lo spazio, la storia, gli uomini, le tradizioni, Bompiani, Milano, I995, p. 7; Scipione Guarracino, Mediterraneo. Immagini, storie e teorie da Omero a Braudel, Bruno Mondadori, Milano, 2007, p. I5.

3. Sanjay Subrahmanyam, Mondi connnessi. La storia oltre l'eurocentrismo (secoli XVI-XVIII), Carocci, Roma, 20I4, p. 27; Idem, "Connected Histories: Notes towards and Reconfiguration of Early Modern Eurasia», Modern Asian Studies, 3 (1997), pp. 735-762; Serge GruZINSKI, «Le monde mêles de la monarchie catholique et autres "Connected Histories"”, Annales. Histoire, Sciences Sociales, I (200I), pp. 85-II7. 
ca medievale. ${ }^{4}$ Tale approccio, ultimamente ripreso da Jaume Dantí l'historia connectada - e da Michael North - mari connessi-, è perfetto per mostrare in che modo il Mediterraneo della piena età moderna fosse connesso attraverso i gruppi mercantili con il resto del mondo. ${ }^{5}$ Il Grande Mare divenne il principale connettore delle diverse storie del globo, integrandosi in maniera via via sempre più forte con il Baltico, l'Atlantico, il Pacifico, l'Oceano indiano e i mari della Cina e del Giappone. Per capire meglio questo fenomeno, North suggerisce di studiare pertanto «le reti che collegavano tra loro i mari e oceani e producevano intrecci tra le diverse sfere commerciali». Il mare, o meglio la storia marittima, diventa in questo modo la chiave di lettura fondamentale, sia per leggere la storia di queste connessioni sia per spiegare la fortuna della storia globale oggi, così come bene ci dice Serge Gruzinski:

Il primato del mondo di mare rispetto a quello di terra può parimenti disturbare, benché sia evidente che i grandi imperi dell'Età moderna, da quello spagnolo a quello portoghese, da quello inglese a quello olandese, sono marittimi. È insomma sui mari, nei passaggi oceanici che si è giocata fino all'inizio del xx secolo la mondializzazione europea. È attraverso l'Atlantico che gli europei emigrano nel Nuovo Mondo, è sulle navi della tratta che gli schiavi africani sono deportati nelle Americhe. È grazie all'autostrada del Pacifico che una quota dell'argento americano si riversa nelle casse cinesi. Il mare rende ragione dell'esplosione della mobilità e incoraggia le circolazioni ai danni delle forme ancestrali di radicamento. ${ }^{6}$

In questa sede, per cercare di proporre ai lettori un esempio possibile di Mediterraneo connesso, utilizzeremo la rete commerciale che univa l'Europa con l'Asia, l'Africa e l'America tra XVII e XVIII secolo,

4. Peregrine Horden e Nicholas Purcell, The Corrupting Sea. A Study of Mediterranean History, Blackwell, Oxford, 2000, pp. I23-172.

5. J. Dantí, coord., Relacions mediterrànies a la Catalunya moderna, Rafael Dalmau, Barcelona, 20I8, p. 5; Michael NorTH, «Mari connessi», nel suo Reti marittime come fattori dell'integrazione europea, Firenze University Press, Firenze, 2019, pp. 5-25.

6. Serge Gruzinski, Abbiamo ancora bisogno della storia? Il senso del passato nel mondo globalizzato, Raffaello Cortina Editore, Milano, 2016, p. I09. 
attraverso lo snodo principale costituito dal porto di Genova. ${ }^{7}$ I vari link che, tramite la diaspora dei mercanti genovesi, furono costruiti nel corso del tempo, costituiscono un modello straordinario per leggere la storia globale delle connessioni mondiali marittime. La capacità dei liguri, che vivevano stretti tra le montagne e il Mediterraneo in una sottile striscia di terra, fin dall'epoca medievale, fu di sapersi spingere via mare in luoghi lontani, creando rotte commerciali sempre più interessanti dal punto di vista economico, spesso anticipando direttrici di traffico mercantile mondiale, poi sfruttate in maniera più organica da altre potenze navali. La storia della diaspora genovese e della progressiva espansione delle connessioni mondiali nel corso dell' età moderna, costituiscono il paradigma interpretativo di questo saggio.

\section{Connessioni genovesi}

A partire dall'epoca di Carlo V la storia di Genova si intreccia in maniera indissolubile con quella della Spagna e della sua espansione fuori dall'Europa. Uomini, navi e capitali furono messi al servizio della potenza asburgica, con evidenti vantaggi economici, finanziari e politici per i genovesi, i quali cominciarono ad essere presenti in tutti i territori controllati dalla monarchia spagnola. Il sistema di simbiosi, definito dagli storici El siglo de los Genoveses, subì nel corso del XVII secolo alcune sostanziali modifiche, dettate dal desiderio di una parte del ceto dirigente della Repubblica di sganciarsi dal sistema di tutela ispanico. Ne nacque nel corso del Seicento un partito, definito "navalista», che propugnava un distacco progressivo dall'alleanza iberica a favore di un rafforzamento del settore marittimo.

Questa svolta politica definita «navalista», compiuta da una parte del ceto dirigente della Repubblica di Genova a partire dagli anni Trenta del XVII secolo, tendente ad ottenere un progressivo sganciamento

7. Francesco Surdich, «I viaggi, i commerci, le colonie: radici locali dell'iniziativa espansionistica», in A. Gibelli e P. Rugafiori, eds., La Liguria, Storia d'Italia. Le regioni dall'Unità ad oggi, Einaudi, Torino, 1994, p. 457. 
dalla «simbiosi spagnola», se da una parte fu velleitaria e fallimentare, come ampiamente dimostrato da Carlo Bitossi, dall'altra ebbe riflessi importanti nel promuovere e stimolare lo sviluppo dell'armamento ligure nella seconda metà del XvII secolo, ${ }^{8}$ dopo qualche decennio di appannamento. La crescita armatoriale coincise, non a caso, con una straordinaria stagione economica, ancorché limitata cronologicamente, che vide i genovesi protagonisti nella tratta degli schiavi in Atlanti$\mathrm{co}^{9}$ e nuovamente presenti - grazie alla firma delle capitolazioni con la Sublime Porta ${ }^{\text {IO }}$ - nel Levante mediterraneo ottomano, dove in pochi anni, sulla scia dei francesi, riversarono una quantità di moneta adulterata tale da mettere in ginocchio la fragile economia turca. Per circa un ventennio la diaspora mondiale dei genovesi - formatasi nel corso dei secoli - si ritrovò ad operare globalmente, quasi vi fosse una sorta di regia, connettendo luoghi e storie molto distanti tra loro, appartenenti a diverse culture: da quella cattolica ibero-americana a quella protestante; ${ }^{\text {II }}$ da quella africana a quella ottomana, con il suo prolungamen-

8. Carlo Bitossi, Il governo dei magnifici. Patriziato e politica a Genova fra Cinque e Seicento, ECIG, Genova, 1990, pp. 214-233; Idem, «Il Genio ligure risvegliato. La potenza navale nel discorso politico genovese del Seicento», in F. Cantù, ed., I linguaggi del potere nell'età barocca, I. Politica e religione, Viella, Roma, 2009, pp. 81-II2; Claudio Costantini, La Repubblica di Genova, Utet, Torino, 1986, pp. 283-315; Idem, "Aspetti della politica navale genovese nel Seicento", Miscellanea storica ligure, II, I (1970), pp. 207-235; Giulio GiacChero, Il Seicento e le Compere di San Giorgio, Sagep, Genova, 1979, pp. 372-376; Luca Lo BAsso, Uomini da remo. Galee e galeotti del Mediterraneo in età moderna, Selene Edizioni, Milano, 2003, pp. 252-266.

9. Marisa Vega Franco, El trafico de esclavos con América (asientos de Grillo y Lomelin, I663-I674), Escuela de Estudios Hispano-Americanos de Sevilla, Sevilla, 1984; Alejandro García MonTón, «Génova y el Atlántico (c. 1650-1680). Emprendedores mediterráneos frente al auge del capitalismo del Norte», tesi di dottorato, European University Institute, Firenze, 2014.

IO. Onorato Pastine, Genova e l'Impero ottomano nel secolo XVII, Società Ligure di Storia Patria, Genova, I952; Carlo Maria Cipolla, Tre storie extra vaganti, Il Mulino, Bologna, 1994, pp. 5I-62.

II. Sul concetto di diaspora: Mathilde Monge e Natalia Muchnir, L'Europe des diasporas. XVIe-XvIIIe siècle, PUF, Paris, 2019, pp. I9-57.

Pedralbes, 39 (20I9), 263-292, ISSN: O2II-9587, DOI: IO.344/PEDRALBES2OI9.39.9 
to cinese, grazie all'intermediazione di Manila. ${ }^{\mathrm{I2}}$ Il tutto unito dal movimento di navi, uomini e merci: non solo mercanti ma, al seguito di questi traffici, persone appartenenti a gruppi sociali molto diversi si ritrovarono ad operare in ogni angolo del globo, secondo le logiche del profitto, in un sistema di accumulazione capitalistica sempre più flessibile - per usare la definizione di Giovanni Arrighi - che determinò il grande successo secentesco dei liguri. ${ }^{13}$ Ma con il 1627 non era cessato il «secolo dei genovesi»? Così come ha ben dimostrato la più recente storiografia, il capitalismo «alla ligure» non era defunto con la famosa quiebra, ma anzi trovò una ripresa spettacolare - per dirla alla Braudel - nel quarto finale del XVII secolo, esplicitatasi proprio grazie alla creazione di una rete di traffici intercontinentali e interculturali.

È stato correttamente fatto notare di recente, da Marco Meriggi e Laura Di Fiore, che l'attenzione storiografica per la nuova World History si è concentrata soprattutto sullo studio delle diaspore commerciali, "ovvero le comunità di mercanti organizzati su base familiare, etnica o religiosa per sostenere gli scambi sulla lunga distanza», caratterizzate dalla «capacità dei loro membri di muoversi agevolmente tra almeno due culture, data la funzione di intermediazione culturale che essi erano chiamati a svolgere». ${ }^{14}$ Tra questi, oltre ai mercanti-impren-

I2. La presenza genovese nelle Filippine non è mai stata oggetto di studio, ma è assai probabile che anche a Manila gli operatori liguri abbiano avuto un ruolo nel corso dell'età moderna, considerato che il porto manilense era il terminale dell'argento americano destinato allo scambio con le merci cinesi. Inoltre, proprio negli anni Sessanta del xvir secolo il Generale delle galere delle Filippine era Sebastián Rayo Doria (Raggio Doria), uomo altolocato sposato con Ana Pestańo de la Cueva, figlia di Marcos, uno dei più importanti mercanti di Manila di quel periodo. Sul ruolo di Manila come porto di interscambio con il mondo cinese si veda il recente lavoro di Juan GIL, Los chinos en Manila (siglos XVI y XVII), Centro Científico e Cultural de Macau, Lisboa, 20II, pp. 65-70; su Rayo Doria: Archivo General de Indias (AGI), Gobierno, Filipinas, leg. 43, n. 17 e n. 23.

I3. Giovanni Arrighi, Il lungo XX secolo. Denaro, potere e le origini del nostro tempo, Net, Milano, 2003, p. I53.

I4. Laura Di Fiore e Marco Meriggi, World History. Le nuove rotte della storia, Laterza, Roma-Bari, 20II, p. 45. Sulle diaspore commerciali: Philip Curtin, Mercan- 
ditori, è opportuno ricordare in questa sede il ruolo importante avuto dai capitani di nave e da tutti i marittimi in generale, uomini tradizionalmente abituati a muoversi tra più culture, ma spesso dimenticati dalla storiografia. Tali considerazioni ci permettono di inserire le vicende qui esposte in una prospettiva di storia globale, nella quale il ruolo esercitato dalla diaspora genovese risulta essere, per qualche decennio, uno dei fulcri attorno a cui ruota la crescita esponenziale del sistema capitalista mondiale tra la fine del secolo Xvir e il secolo XviII.

La stagione dei «navalisti», come già accennato, si aprì nella seconda metà degli anni Trenta del Seicento. Seppur indirettamente, alcuni polemisti - tra cui Andrea Spinola - già negli anni Venti avevano fornito materiale utile per suggerire al ceto di governo della Repubblica un ritorno al mare in grande stile, slegato dalla tradizionale simbiosi ispanica, messa in dubbio dal comportamento tenuto dagli Austrias durante la guerra savoina del I625.5 Pochi anni dopo, la politica «navalista» maturò ulteriormente nel clima della guerra che si era aperta tra la Francia e la Spagna e trovò a Genova alcuni importanti membri di spicco del patriziato decisi a farla propria. La partita risolutiva si giocò tra il I637 e il I638, quando, dapprima, si decretò che Genova fosse un Regno con a capo la Vergine Maria e, per secondo, allorché si procedette all'armamento delle prime galee di libertà, il tutto favorito dal doge Agostino Pallavicini, un antispagnolo convinto. ${ }^{16}$

ti. Commercio e cultura dall'Antichità al XIX secolo, Laterza, Roma-Bari, I999; Francesca Trivellato, Il commercio interculturale. La diaspora sefardita, Livorno e i traffici globali in età moderna, Viella, Roma, 2or6; ma soprattutto le preziose pagine introduttive della stessa Trivellato al volume F. Trivellato, L. Halevi e C. Antunes, eds., Religion and Trade. Cross-Cultural Exchanges in World History, I0oo-I9oo, Oxford University Press, Oxford, 20I4, pp. I-24. Recentemente è stato studiato il rapporto tra la storia marittima e la storia globale e per questo si veda: Patrick Manning, «Global History and Maritime History», International Journal of Maritime History, XXV, I (2013), pp. I-22.

I5. D. Puncuh, ed., Storia di Genova, Società Ligure di Storia Patria, Genova, 2003, pp. 435-44I.

I6. Bitossi, Il Genio ligure risvegliato, p. 89. 
Nel 1638 si diede avvio alla svolta "navalista» grazie all'azione dei fratelli Francesco Maria e Galeazzo Giustiniani, i quali armarono la prima galea soltanto con rematori liberi e volontari, per compiere viaggi in Sicilia e in corso; da quel momento e fino al I684, in seno al governo genovese, fu tutto un susseguirsi di proposte, calcoli, relazioni attorno all'armamento delle galere di libertà. ${ }^{17}$ Nonostante i buoni propositi e anche qualche buon risultato, soprattutto nelle primissime campagne, l'esperimento delle nuove galee si rivelò fallimentare e velleitario perché, colpendo in particolare gli interessi degli assentisti (asentistas) delle galee spagnole, contribuì a complicare i rapporti simbiotici tra questi ultimi e la corte di Spagna, e di conseguenza alterò le relazioni tra il gruppo di genovesi residenti a Madrid e quello al potere in quel momento a Genova, in una fase politica delicata, complicata dalla guerra contro la Francia. Se dunque politicamente l'avventura navalista non ebbe grandi risultati nella fase delicata degli anni Trenta e Quaranta del secolo XviI, è indubbio che per converso fu rivitalizzata in un primo momento negli anni Cinquanta, quando il governo della Repubblica decise di istituire nel 1655 il Magistrato del Nuovo Armamento con l'incarico di organizzare un convoglio pubblico sulla rotta tra la Spagna e Genova, per meglio proteggere e favorire non solo il recupero dei capitali investiti dai genovesi nel mondo iberico ma anche i regolari traffici commerciali che tradizionalmente si tenevano su quell'itinerario. ${ }^{18} \mathrm{La}$ svolta era stata suggerita proprio dall'esigenza di rafforzare il trasporto dell' argento spagnolo, considerato che le sole galee pubbliche nel biennio I652-53 avevano incassato noli per più di 890.000 lire. La nascita del pubblico convoglio e del Nuovo Armamento ebbe un effetto positivo sul mondo dello shipping genovese solamente a partire dagli

17. Lo Basso, Uomini da remo, pp. 252-266.

I8. Gian Carlo CalCagno, "Armamento pubblico e magistrature marittime a Genova nei secoli Xvi e Xviı», nel suo La Storia dei genovesi, Tipolitografia Sorriso Francescano, Genova, 1985, VI, pp. 2I-38; Idem, «La navigazione convogliata a Genova nella seconda metà del Seicento", nel suo Guerra e commercio nell'evoluzione della marina genovese tra XV e XVII secolo, Istituto di storia moderna e contemporanea, Genova, 1973, pp. 267-392. 
anni Sessanta, dopo la fine della guerra franco-spagnola e dopo che la stessa Genova superò definitivamente la crisi epidemica del I656-I657. Il settore armatoriale ligure, che da qualche decennio sonnecchiava aggrappato al medio e piccolo cabotaggio, trovò un nuovo e vitale slancio, definito «spettacolare» da Fernand Braudel: Genova non aveva dunque «lasciato la preda». Ma quali furono le modalità di questo sorprendente rilancio? ${ }^{19}$

La politica navalista, oltre all'esperimento delle galee di libertà e all'istituzione del pubblico convoglio, si era manifestata in alcuni tentativi di espansione fuori dal Mediterraneo. Il primo esperimento, quello più noto storiograficamente, è quello della Compagnia Genovese delle Indie Orientali, ${ }^{20}$ costituita nel I647 su iniziativa dell'ambasciatore olandese a Genova Henrik Muilman, desideroso di trovare uno sfogo commerciale per tutti i mercanti olandesi esclusi dagli affari della VOC. Dopo essersi dotata di due navi costruite a Texel ed equipaggiate soprattutto con personale olandese, la compagnia organizzò la prima spedizione verso l'Asia. Le due navi, salpate il 3 marzo I648, erano ufficialmente dirette in Giappone, anche se, come ha evidenziato Subrahmanyam, in realtà la loro vera destinazione era interna ai territori controllati dagli olandesi o dai portoghesi, ${ }^{21}$ e in effetti nel marzo del 1649

19. Fernand Braudel, Il secondo Rinascimento. Due secoli e tre Italie, Einaudi, Torino, 1986, p. 79; Luca Lo BAsso, Capitani, corsari e armatori. I mestieri e le culture del mare dalla tratta degli schiavi a Garibaldi, Città del Silenzio, Novi Ligure, 20II, pp. 17-39.

20. Sanjay Subrahmanyam, "On the Significance of Gadflies: the Genoese East India Company of the I640s", The Journal of European Economic History, 3 (1988), pp. 559-581; Thomas KIRK, "A little Country in a World Empire», The Journal of European Economic History, 2 (1996), pp. 407-42I.

2I. Danilo Presotto, «Da Genova alle Indie alla metà del Seicento. Un singolare contratto di arruolamento marittimo", Atti della Società Ligure di Storia Patria, IX ns. (I969), pp. 69-9I; Costantini, La Repubblica di Genova, pp. 315-32I; Luigi Tommaso Belgrano, «La compagnia delle Indie e Tommaso Skynner», Giornale Ligustico, II (I875), pp. I2I-I36; Idem, «Un documento circa la navigazione dei genovesi alle Indie», Giornale Ligustico, II (1875), pp. 254-257; Idem, «Opuscoli di Benedetto Scotto gentiluomo genovese circa un progetto di navigazione pel settentrione alla China 
i due vascelli diedero fondo alle ancore davanti a Sumatra. Nel contempo, però, i portoghesi, all'epoca ribellatisi agli Asburgo di Spagna, concordarono con i vertici della VOC l'eliminazione della concorrenza genovese, considerata molto pericolosa, poiché legata al comune nemico spagnolo. I portoghesi, con tutta evidenza, non avevano coscienza del fatto che la Compagnia ligure era voluta da una fazione politica interna alla Repubblica, desiderosa proprio di sganciarsi il più possibile dall'alleanza asburgica. Entro un mese dall'arrivo delle navi in Asia esse furono sequestrate assieme al loro carico; i marinai olandesi furono arruolati a forza sulle navi della VOC e i genovesi presenti a bordo furono rispediti in Europa. A Genova nessuno sospettava l'opposizione del Portogallo all'impresa asiatica, ma, anzi, proprio in quegli anni i membri del partito navalista speravano di trovare un'alleanza con i lusitani, logorati dalla guerra in patria contro la Spagna, con pochi veri alleati e con necessità di ottenere navi e capitali. ${ }^{22} \mathrm{~A}$ riprova di ciò, nel I647 la Repubblica nominò un console a Lisbona: dapprima, Francesco Bresciani, e poco dopo Giovanni Gerolamo Ghersi, ${ }^{23}$ uno dei più importanti mercanti liguri residenti nella capitale lusitana. Negli stessi mesi, alcuni genovesi residenti in Portogallo stavano tentando di arma-

ed alle Indie Orientali editi nel principio del secolo xvir e di presente ripubblicati dal socio Luigi Tommaso Belgrano», Atti della Società Ligure di Storia Patria, V (I867), pp. 273-355. I capitoli della compagnia del 22 gennaio I648 e numerosi altri documenti si trovano in Archivio Saluzzo-Sopranis di Genova (ASSG), leg. I4I.

22. Nunziatella Alessandrini ed Antonella Viola, «Genovesi e fiorentini in Portogallo: reti commerciali e strategie politico-diplomatiche (I650-1700)", Mediterranea ricerche storiche, 28 (2013), pp. 295-322; si veda anche la lettera del capitano di una delle navi della compagnia, Costantino Caffarello, del 3 aprile i66o. Archivio di Stato di Genova (ASG), Archivio Segreto, Lettere Consoli Portogallo, leg. 2659. Le istruzioni al capitano Caffarello, comandante del galeone S. Giuseppe, e al capitano Gio. Agostino Germano, comandante della nave S. Anna, per il viaggio in Brasile, del 27 gennaio I660 si trovano in ASSG, leg. I4I.

23. Nunziatella AlessandrinI, "Consoli Genovesi a Lisbona (I650-I700 ca.)», in M. Aglietti, M. Herrero Sánchez e F. Zamora Rodríguez, coords., Los Cónsules de extranjeros en la Edad Moderna y a principios de la Edad Contemporánea, Ediciones Doce Calles, Madrid, 2013, p. 205. 
re una flotta destinata in India senza il permesso reale. Secondo una nota di protesta, trasmessa al Senato della Repubblica, gli interessati erano: Giovanni Laviosa, Giovanni Filippo Cattaneo, Nicolò D’Amico, Ugo Fieschi e Scipione Bonvicino. ${ }^{24}$ Un anno più tardi (Io marzo I649) altri commercianti genovesi di Lisbona, Nicolao Micone, Francesco Carrega e Giovanni Girolamo Ghersi, parteciparono con capitale proprio alla costituzione della «Companhia Geral do Brasil»" ${ }^{25}$, che ebbe una certa attività fino agli anni Sessanta. ${ }^{26}$

Nel I653, inoltre, venne costituita la Compagnia Marittima di San Giorgio, con un prestito dell'omonimo Banco; essa ereditava ed estendeva i privilegi e le prerogative della precedente Compagnia, alcuni membri, come Ugo Fieschi e Gio. Bernardo Veneroso, ma non le navi - San Bernardo e San Giovanni Battista -, che, secondo la documentazione analizzata da Subrahmanyam, furono vendute alla VOC. ${ }^{27}$

La nuova compagnia di San Giorgio riuscì ad ottenere il diritto di inviare navi in Brasile, suscitando però invidie da parte di alcuni gruppi di mercanti portoghesi. Ad ogni modo, tra I655 e il I657 furono organizzati almeno due viaggi, con un terzo viaggio svoltosi molto probabilmente tra il I662 e il I663. ${ }^{28}$ E credibile che tra il I657 e il I662 le stesse

24. ASG, Archivio Segreto, Secretorum, leg. 1572.

25. Alessandrini-Viola, Genovesi e fiorentini in Portogallo, p. 303.

26. Uno dei finanziatori dell'avventura brasiliana genovese fu Stefano Pallavicini. Cfr. Leonor Freire Costa, O trasporte no Atlântico e a Companhhia Geral do Comércio do Brasil (I580-I663), 2 vols., Comissão Nacional para as Comemoraçóes dos Descobrimentos Portugueses, Lisboa, 2002, I, pp. 553-559.

27. ASG, Banco di San Giorgio, Cancellieri, leg. 48I.

28. Secondo una testimonianza di quattro marinai imbarcati sul galeone $S$. Giuseppe, la spedizione in Brasile si era conclusa nella primavera del I663. Oltre al $S$. Giuseppe, comandato dal capitano Bartolomeo Petropoli, che aveva sostituito il capitano Caffarello, vi era la nave $S$. Anna, da 32 cannoni, del capitano Germano. ASSG, leg. I4I; 8 giugno I663; l'inventario della nave $S$. Anna del 23 ottobre I659; l'inventario del S. Giuseppe del 24 gennaio I66o. Sulla Compagnia di S. Giorgio si rimanda anche a Giorgio Tosco, «La Compagnia Genovese delle Indie Orientali e i rapporti fra Genova e le Province Unite nel Seicento», tesi di laurea, Università di Pisa, a.a. 201320I4, pp. I38-I54. 
navi della Compagnia abbiano seguito anche altre rotte europee, così come si ricava da un atto notarile del $1659 .{ }^{29}$ Con tutta evidenza, l'attività della Compagnia cessò definitivamente nel 1665, quando ormai lo sguardo degli operatori marittimi genovesi era rivolto al nuovo asse commerciale creatosi tra la tratta degli schiavi e il traffico levantino. ${ }^{30}$

Nei primi anni Sessanta, grazie alla politica navalista, si erano riviste in mare navi di grandi dimensioni costruite sulle spiagge nelle immediate vicinanze di Genova e finanziate dai più importanti membri dell'aristocrazia cittadina, nuovamente attratti dal settore dello shipping; tra queste ricordiamo la Santa Rosa, costruita ad Arenzano, la Sant'Antonio del capitano Pietro Ferrari, la Nostra Signora del Rosario del capitano Michelangelo Rosso, la Nostra Signora di Loreto del capitano Gio. Agostino Germano, la S. Tomaso di Villanova del capitano Nicolò Lanata e la Nostra Signora dell'Apparizione e San Pietro del capitano Pietro Bianchi (nei documenti anche Bianco, Blancus in latino, Blanco alla spagnola). Quest'ultima nave, dopo aver navigato sulla rotta Genova-Cadice-Lisbona per lunghi anni, fu venduta nel porto gaditano nel I67I, proprio mentre sulla spiaggia di Savona iniziavano i lavori di costruzione del nuovo vascello del medesimo capitano Bianchi, denominato come il precedente, dei quali si possiede una corposa documentazione conservata nell'archivio di uno dei più importanti caratisti $\mathrm{i}^{3 \mathrm{I}} \mathrm{e}$ che dunque si presta a ricoprire in questo contesto il ruolo di testimonial dell'intero settore marittimo. Seguendo la vita di questo bastimento è possibile perciò tracciare il modello seguito per il fruttuoso riarmo navale ligure del XVII secolo. All'epoca queste grandi navi avevano costi di costruzione e di gestione molto elevati e perciò necessitavano di un cospicuo numero di soci/caratisti, così come si

29. ASG, Notai giudiziari, Gio. Gerolamo Camere, leg. 23II.

30. ASG, Archivio Segreto, Giunta del Traffico, leg. IoI5.

3I. Archivio Durazzo Giustiniani di Genova (ADGG), Archivio Sauli, leg. 1374, Libro della fabrica della nave Apparizione di Nostra Signora e S. Pietro. Sulle vicende costruttive di questa nave, Luca Lo Basso, A vela e a remi. Navigazione, guerra e schiavitù nel Mediterraneo (secc. XVI-XVIII), Philobiblon Edizioni, Ventimiglia, 2004, pp. 68-82. 
vede proprio nel caso della nave Nostra Signora dell'Apparizione. Il bastimento era diviso in ventiquattro quote (carati), acquistabili in numero variabile a seconda delle opportunità. ${ }^{32}$ Il prezzo di ogni singola quota era dato dall'ammontare del costo della nave finita (scafo, manodopera, attrezzature e cannoni), diviso ventiquattro. In ogni momento l'azionista poteva cedere le proprie quote e, di conseguenza, al suo posto poteva eventualmente subentrare un nuovo caratista. Nel caso della nave del capitano Bianchi, i finanziatori erano diciotto, tra cui segnaliamo, oltre al capitano Pietro Bianchi, Francesco Maria Sauli - molto attivo nella gestione dell'asiento degli schiavi -, Pietro Durazzo - uno dei protagonisti dell'avventura genovese in Levante - e Vincenzo Spinola, attivo in quegli anni nel commercio di artiglierie. ${ }^{33}$ I lavori di costruzione, iniziati nel settembre I67I, si conclusero il primo ottobre I672, sotto la direzione di Ambrogio Fava di Varazze, uno dei più quotati maestri d'ascia dell'epoca. La nave, dopo aver completato l'allestimento nel porto di Savona, fu consegnata per il viaggio inaugurale nel maggio del 1673 ed il costo complessivo fu di lire genovesi I34.55I, compreso il prezzo dei quaranta cannoni, forniti per la maggior parte da Vincenzo Spinola.

La nave fu messa in servizio nel corso del I673, trasportando riso da Genova verso Lisbona, e a seguito di questo viaggio il Sauli, il 4 maggio I674, registrò sul suo libro giornale gli utili pari a lire 845 . Inoltre, nel corso dell'anno, il futuro doge della Repubblica incassò altre 1333 lire ricavate dalla vendita della vecchia nave del capitano Pietro Bianchi, venduta a Cadice l'anno precedente. ${ }^{34}$ Il secondo viaggio del I674 cominciò il 20 maggio. Dopo una breve tappa livornese, la Nostra Signora dell'Apparizione ripartì verso Cadice e Lisbona, tradizionale rotta di questo tipo di navi. L'II giugno si trovava a Finale per caricare diversi

32. Luciana GatTI, Navi e cantieri della Repubblica di Genova (secoli XVI-XVIII), Brigati, Genova, 1999, pp. 85-I24; Gian Domenico Peri, Il Negotiante, Gio. Giacomo Herz, Venezia, I673, III, pp. 26-42; Carlo TARga, Ponderazioni sopra la contrattazione marittima, Stamperia della Libertà, Genova, 1803, pp. 15-19.

33. ADGG, Archivio Sauli, leg. I377, 5 aprile 1675.

34. ADGG, Archivio Sauli, leg. 908, 4 maggio I674 e I2 settembre I674. 
generi alimentari per il consumo di bordo e il 25 dello stesso mese toccò Alicante e poi, il I4 luglio, Cadice; infine giunse l'8 agosto a Lisbona. Nel porto lusitano il capitano Bianchi si trattenne fino al 24 settembre, quando riprese il mare. Fece scalo il 26 ancora a Cadice, il i8 ottobre giunse ad Almeria e il 24 si ritrovò alla fonda davanti ad Alicante. Il 24 novembre la Nostra Signora dell'Apparizione entrava sana e salva nel porto di Genova. ${ }^{35}$ Negli anni seguenti la nave continuò a fare la spola fra Genova, Napoli, Palermo e i porti spagnoli, con saltuarie puntate a Lisbona. Tra il I68I e il I682 vi fu un sostanzioso rimpasto dei caratisti. Il nuovo comandante divenne Giacomo Maria Viano. Nell'aprile del i684 la Nostra Signora dell'Apparizione venne presa a noleggio dalla Serenissima per l'imminente nuova guerra al Turco, ${ }^{36}$ seguendo le vicissitudini di numerosi vascelli genovesi. L'attacco francese a Genova ritardò la partenza di un anno, che finalmente avvenne nel corso del I686. La nave, agli ordini del capitano Viano, si unì così alla nuova Armata grossa veneziana assieme ad altre unità liguri: Nostra Signora del Carmine, S. Maria, SS. Concezione, SS. Annunziata. Il Io aprile 1687 risultavano in servizio presso l'Armata veneta due navi genovesi: una era la Nostra Signora dell'Apparizione, l'altra era la Santissima Concezione. ${ }^{37}$ Infine, nella primavera dell'anno seguente la Nostra Signora dell'Apparizione subì notevoli danni agli alberi a causa di una burrasca che la colse nel Golfo di Lepanto, mentre era comandata da Filippo Viano, figlio di Giacomo Maria. L'ultima notizia certa riguardante la nostra nave è del gennaio $1690 .{ }^{38}$

In quegli anni, d'altronde, molte di queste navi servivano, oltre che al soldo dei veneziani, nella carrera de Indias, così come si ricava

35. Nei manifesti di carico dei viaggi di ritorno si trovano soprattutto casse e sacchetti d'argento in barre o in monete.

36. Archivio di Stato di Venezia (ASV), Senato Mar, leg. 65I, 29 aprile I684. Sull'armata veneziana rimando al fondamentale libro di Guido Candiani, I vascelli della Serenissima. Guerra, politica e costruzioni navali a Venezia in età moderna, I650I720, Istituto veneto di Scienze, Lettere ed Arti, Venezia, 2009.

37. ASV, Senato Mar, leg. 668, allegato del Io aprile I687.

38. ASV, Senato Mar, leg. 683. 
dai numerosi documenti conservati nell'Archivo General de Indias di Siviglia. Una di queste unità di costruzione genovese, ad esempio, era la Nostra Signora del Carmelo, che nel 1668, dopo aver servito nell'Armada, era passata al trasporto merci sulla rotta per le Indie. ${ }^{39}$ Dai registri delle navi in partenza per l'America si evince che nel I67I era partita per Caracas proprio una nave genovese chiamata Nuestra Señora de la Aparicion y San Pedro, comandata in quella circostanza da Jacinto Serrano e che con tutta evidenza era la stessa venduta dal capitano Bianco a Cadice all'inizio del $\mathrm{I} 67 \mathrm{I} .{ }^{40} \mathrm{La}$ medesima nave nel $\mathrm{I} 673$ venne registrata per un nuovo viaggio a Caracas, questa volta comandata da Francisco Blanco, parente stretto del capitano Pietro, residente a Sanlúcar de Barrameda e naturalizzato spagnolo..$^{41} \mathrm{D}$ 'altra parte, qualche anno più tardi, nel I683, ritroviamo impegnato nella traversata dell'Atlantico anche lo stesso capitano Pietro Bianchi al comando del vascello Nuestra Señora del Rosario y San Joseph, questa volta di «fabrica olandesa».42 $\mathrm{Ed}$ ancora, qualche anno dopo, a riprova della presenza di navi e comandanti liguri sulla rotta delle Americhe, nel I699 troviamo, sempre in direzione Caracas, una Nostra Signora dell'Apparizione e Sant'Antonio da Padova "de fabrica Genovesa», della portata di 406 tonnellate, amministrata da Agostino de Castro. ${ }^{43}$ Per non parlare di quei vascelli e di quei capitani facenti parte del giro dell'asiento de los negros, come Juan Francisco Garibaldo, ${ }^{44}$ comandante della Santa

39. AGI, Indiferente General, leg. 78I, 24 marzo I668.

40. AGI, Contratación, leg. 2900, c. 246r, I5 luglio I67I.

4I. AGI, Contratación, leg. I224, n. I/reg. 5; altre notizie si trovano in Contratación, leg. I223, n. Io.

42. AGI, Contratación, leg. 2900, c. 269 r.

43. Ibidem, c. 3Ior.

44. Il capitano Garibaldi tra il I67I ed il I672 trasportò 1563 schiavi tra i porti di Curaçao, Portobelo, Veracruz e Cartagena sempre a bordo della nave Santa Cruz (AGI, Contaduría, leg. 263 e I4I8; Contratación, leg. 2900, c.244V, 29 dicembre I670, «El navío nombrato Santa Cruz uno de los asientos de Domingo Grillo y Compañía. Capitan Juan Francisco Garibaldo que fue a la isla de Curazao a rescate de negros»). Il medesimo capitano era partito per l'America nel I667, così come si ricava dal suo 
Cruz, e Franco Gallesio, comandante della S. Nicolò da Tolentino, ${ }^{45}$ registrati in più occasioni sulla rotta tra l'Angola, Portobelo, Cartagena de Indias e Curaçao.

Questa nuova attenzione verso l'armamento marittimo su vasta scala fu generata, da una parte, come detto, dalla svolta navalista della Repubblica, ma dall'altra fu figlia anche di una politica economica intrapresa da una nuova generazione di operatori genovesi. Alcuni di questi, tra il I662 e il I665, riuscirono a stipulare, come è noto, l'asiento degli schiavi, che permise loro di gestire direttamente una larga fetta del cosiddetto "commercio triangolare» almeno fino alla metà degli anni Settanta del XVII secolo. L'asiento Grillo-Lomellini non ha goduto per lungo tempo di un'attenzione storiografica, fatta eccezione per i riferimenti nel pioneristico lavoro di Scelle, ${ }^{46}$ dei primi del Novecento, per alcune stimolanti anticipazioni di Roberto Lopez ${ }^{47}$ e di Fernand Braudel ${ }^{48}$ e finalmente per la monografia del 1984 della già citata Vega Franco, mentre più recentemente è tornato ad interessare gli storici

passaporto rilasciato il is febbraio a Siviglia dalla Casa de la Contratación (AGI, Contratación, leg. 5435, n. 2, r. 3 ).

45. Il I $^{\circ}$ settembre 167 I giunse a Caracas la nave La Concordia Dorada con un carico di 259 schiavi comandata da Franco Galesio, il quale dichiarò di essere partito da Curaçao il primo agosto I67I e di non aver avuto decessi durante il viaggio. A Caracas gli schiavi furono consegnati al factor don Juan Antonio Blanco (AGI, Contaduría, leg. 263). Due anni prima il medesimo comandante aveva scaricato schiavi nel porto di Cartagena, dopo averli imbarcati a Curaçao e Portobelo sulla nave $S$. Nicolò da Tolentino (AGI, Contaduría, leg. 265). Più in generale, il medesimo capitano, tra il 1669 ed il I672, trasportò I205 schiavi nei porti di Veracruz e Portobelo (AGI, Contaduría, leg. I4I8).

46. Georges SCELle, Histoire politique de la traite négrière aux Indes de Castille, Librarie de la Société du Recueil, Paris, 1906.

47. Roberto Sabatino Lopez, «Il predominio economico dei genovesi nella Monarchia spagnola», Giornale Storico e Letterario della Liguria, XII (1936), pp. 65-74; Idem, "Market Expansion: the Case of Genoa», Journal of Economic History, 24/2 (1964), pp. 445-464.

48. Fernand Braudel, "Le siècle des Génois s'achève-t-il en I627?», nel suo $A u$ tour de la Méditerranée, Éditions de Fallois, Paris, 1996, pp. 433-446. 
spagnoli, come Manuel Herrero Sánchez, Igor Pérez Tostado ${ }^{49}$ e, soprattutto, Alejandro García Montón. ${ }^{50}$

L'asiento de los negros firmato da Ambrogio Lomellini e Domenico Grillo il 5 luglio 1662 rappresentò una svolta definitiva nel sistema organizzativo della tratta degli schiavi atlantica, perché questo accordo spezzò definitivamente il protezionismo commerciale spagnolo sulla carrera de Indias, permettendo ai genovesi - che difatti furono successivamente osteggiati a Madrid - di far entrare nel traffico commerciale con le colonie spagnole anche gli operatori olandesi ed ingle$s i$, tradizionalmente nemici della corona asburgica. Si trattava di una sorta di «laboratorio organizzativo», nuovo rispetto al sistema vigente nel mondo spagnolo, all'interno di uno spazio atlantico che però da tempo vedeva impegnati anche altri attori politici ed economici. ${ }^{\text {sI }} \mathrm{Il}$ sistema della tratta spagnola, difatti, era stato gestito fino al I640 dai portoghesi, ${ }^{52}$ ai quali dopo tale data erano subentrati altri operatori, organizzati attorno alle nuove basi schiavistiche di Curaçao e delle Barbados. Tra gli anni Quaranta e Cinquanta del Xvir secolo, infatti, gli olandesi avevano rafforzato la loro presenza nell'area caraibica, tra-

49. Manuel Herrero Sanchez e Igor Pérez Tostado, "Conectores del mundo atlántico: los irlandeses en la red comercial internacional de los Grillo y Lomelín», in I. Pérez Tostado e E. García Hernán, coords., Irlanda y el Atlántico ibérico. Movilidad, participación e intercambio cultural (I580-I823), Albatros Ediciones, Valencia, 20IO, pp. 307-32I.

50. Alejandro García Montón, «Trayectorias individuales durante la quiebra del sistema hispano-genovés: Domingo Grillo (I617-1687)», in M. Herrero Sánchez, Y. Rocío Ben Yessef Garfia, C. Bitossi e D. Puncuh, coords., Génova y la Monarquía Hispánica (I528-I7I3), 2 vols., Società Ligure di Storia Patria, Genova, 20II, I, pp. 367384; Idem, "Implicaciones del mundo (trans)atlántico entre la aristocracia genovesa. Algunas consideraciones en torno a la segunda mitad del siglo XviI", in C. Bravo Lozano e R. Quiros Rosado, coords., En tierra de confluencias. Italia y la Monarquía de España, siglos XVI-XVIII, Albatros Ediciones, Valencia, 2013.

5i. García Montón, Génova y el Atlántico (c. I650-I680), pp. I53-I55.

52. Arlindo Manuel Caldeira, Escravos e traficantes no Império português. $O$ comércio negreiro português no Atlântico durante os séculos XV a XIX, A Esfera dos Livros, Lisboa, 2013. 
sformando proprio l'isola di Curaçao nella nuova base di smercio degli schiavi africani anche nelle colonie spagnole, grazie anche ad una politica facilitata dal rilascio delle licenze da parte della WIC olandese. ${ }^{33}$ In contemporanea, inoltre, anche gli inglesi avevano provato ad inserirsi nell'affare, tramite tentativi d'accordo con la corte di Madrid, ma mai incisivi, a causa di una presenza in Africa ancora incerta rispetto a quella olandese. ${ }^{54}$ Insomma, già prima dell'arrivo dei genovesi, quello che forse erroneamente viene definito monopolio del traffico degli schiavi era stato interrotto e su questo solco i nuovi operatori liguri cercarono e trovarono solide alleanze proprio con le compagnie commerciali nordiche. La novità del 1662 fu che la Corona, come aveva già fatto nel primo Cinquecento, rilasciò il permesso alla sola compagnia genovese, un unico privilegio che avrebbe costituito il modello al quale si rifecero anche gli asientos successivi stipulati tra la fine del xvir e l'inizio del xviII secolo. 5 Grazie alla firma di questo contratto, i genovesi, all'epoca nuovamente tornati importanti nel sistema dei prestiti alla Corona, ${ }^{56}$ riuscirono ad inserirsi appieno nel traffico oceanico con uomini e mezzi propri, proponendo anche una

53. Johannes Postma, The Dutch in the Atlantic Slave Trade I600-18I5, Cambridge University Press, Cambridge, 1990, pp. 26-55.

54. Filipa Ribeira da Silva, Dutch and Portuguese in Western Africa. Empires, Merchants and Atlantic System I580-1674, Brill, Leiden-Boston, 20II, pp. 213-247. Uno dei tentativi di accordo tra Madrid e Londra è datato Is dicembre I667 (AGI, Indiferente General, leg. 780).

55. Reyes Fernández Durán, La Corona española y el tráfico de negros. Del monopolio al libre comercio, Ecobook, Madrid, 20II; Fernando Serrano Mangas, La encrucijada portuguesa: esplendor y quiebra de la unión ibérica en las Indias de Castilla, I600-1668, Diputación de Badajoz, Badajoz, I994, p. 71; sull'arrivo massiccio di operatori commerciali stranieri si vedano: Lutgardo GaRCía Fuentes, El comercio español con América, I650-I700, Escuela de Estudios Hispano-Americanos, Sevilla, I980, p. 307 e Antonio García-Baquero González, La Carrera de Indias: suma de la contratación y océano de negocios, Sociedad Estatal para la Exposición Universal Sevilla, Sevilla, I992, pp. 88-II5.

56. Carmen Sanz Ayan, Los banqueros de Carlos II, Universidad de Valladolid, Valladolid, 1989. 
svolta tecnica nell'adozione di galeoni di più elevato tonnellaggio rispetto al passato. ${ }^{57}$

Il sistema messo in atto da Grillo e Lomellini si basava inizialmente sul trasporto degli schiavi da parte degli olandesi a Curaçao, dove l'agente Francesco Maria Compiano smistava «l'oro nero» verso porti spagnoli come Vera Cruz, Portobelo di Panama, Caracas, Cartagena de Indias, Cumanà. In seconda battuta, come detto, si usava «la via inglese», tramite Giamaica e Barbados, dove agiva l'irlandese Ricardo Fuit. Questi era uno dei tanti uomini della rete commerciale di Domenico Grillo e Ambrogio Lomellini, mentre altri ancora erano il noto empolese Francesco Feroni, ${ }^{58}$ Alessandro Bosco e il bergamasco Ottavio Tensini, tutti residenti ad Amsterdam. ${ }^{99}$ Sappiamo con certezza che proprio grazie ad Alessandro Bosco, già il Is settembre I662, i due assentisti firmarono un contratto con la WIC che prevedeva l'invio annuale di 2000 schiavi da portarsi a Curaçao. Dai contratti stipulati con gli olandesi e con gli inglesi scopriamo che i due genovesi si erano spinti ben oltre gli accordi sottoscritti con la Corona. Secondo tali patti, i due assentisti avrebbero dovuto trasportare 3500 schiavi all'anno, mentre secondo gli accordi stipulati con gli olandesi e con gli inglesi il numero degli schiavi da trasportare sarebbe stato di 9200 . In sostanza, ben 5700 schiavi da vendere fuori dal contratto. ${ }^{60}$ Come accennato, però, queste violazioni giocarono un ruolo fondamentale nell'ostacola-

57. Fernando Serrano Mangas, Función y evolución del galeón en la Carrera de Indias, Editorial MAPFRE, Madrid, I992; AGI, Indiferente General, leg. 2703; AGI, Contaduría, leg. 562, 3 luglio I663 e 27 gennaio I664.

58. Paola Benigni, "Francesco Feroni, empolese, negoziante in Amsterdam", Rassegna degli Archivi di Stato, 48/3 (1988), pp. 488-517; Hans Cools, «Francesco Feroni, intermediario in cereali, schiavi e opere d'arte», Quaderni Storici, I22/2 (2006), pp. 353-365; Archivio di Stato di Firenze (ASF), Mediceo del Principato, leg. 4260 e leg. $426 \mathrm{I}$.

59. M. Keblusek e B. V. Noldus, eds., Double Agents. Cultural and Political Brokerage in Early Modern Europe, Brill, Leiden-Boston, 20II, p. IO2.

6o. Vega Franco, El tráfico de esclavos, pp. 203-217; AGI, Contaduría, leg. 2526I-262-263-264A-264B-265; Contratación, leg. 4898; Indiferente General, leg. $2830-283 \mathrm{I}-2832-2833-2834-2835-2836$. 
re il funzionamento dell'asiento da parte di molti importanti membri della corte di Madrid. Con lo scoppio della guerra anglo-olandese i due assentisti genovesi furono costretti a fare da soli e per questo chiesero uno sforzo ad altri membri del patriziato della Superba, come Francesco Maria Sauli, Carlo Imperiale e Marc'Antonio Grillo, nipote di Domenico e futuro Grande di Spagna e marchese di Clarafuente. ${ }^{61}$ Proprio la partecipazione di Francesco Maria Sauli risulta essere fondamentale nella gestione marittima dell'asiento, grazie al ruolo che il futuro doge della Repubblica aveva nel contesto dello shipping e nel mondo delle assicurazioni. Senza entrare nel dettaglio del funzionamento dell'asiento, per il quale rimando ai lavori della Vega Franco e di García Mónton, vorrei in questa sede evidenziare alcuni passaggi chiave dell'organizzazione schiavistica messa in piedi dai genovesi e come questi si servirono delle loro conoscenze marittime per gestire in maniera virtuosa il traffico atlantico. ${ }^{62}$ Agli inizi del I666, quando l'asiento procedeva ancora un po' a rilento, l'intervento del Sauli dissipò le nubi che lo circondavano, così come si ricava dalla lettera del 4 maggio in cui Francesco Maria «sentiva particolar gusto che il negotio de negri camminasse felicemente». La notizia era confermata nella lettera del Io maggio scritta da Carlo Imperiale a Domenico Grillo in cui si sottolineava che tramite il Feroni erano stati acquistati 600 neri d'Angola. Il 5 giugno, inoltre, Ambrogio Lomellini scrisse che erano pronte due nuove navi dell'asiento, che avrebbero servito come Capitana e Almiranta della flotta delle Indie, oltre alla Capitana Real, che si sarebbe aggiunta presto alle prime due: tutte navi fornite dagli stessi assentisti, i quali avevano insistito a lungo per introdurre galeoni più grandi, da

6I. ADGG, Archivio Sauli, leg. I465-I466-I587 e Archivio Pallavicini, ramo cadetto, leg. 2I9, copialettere di Carlo Imperiale (I664-I668).

62. Sulla tratta degli schiavi esiste una bibliografia sterminata e in questa sede mi limito a far riferimento al volume di sintesi di Herbert KLeIn, Il commercio atlantico degli schiavi, Carocci, Roma, 2oI4. Sugli aspetti marittimi del traffico schiavistico si veda Henriqueta Vila VILAR, "Aspectos marítimos del comercio de esclavos con Hispanoamérica en el siglo XviI", nel suo Aspectos sociales en América colonial. De extranjeros, contrabando y esclavos, Instituto Caro y Cuervo, Bogotá, 200I, pp. I49-I73. 
500 tonnellate, al posto di quelli in uso in quel momento che al massimo erano da 200 tonnellate. Il mese successivo, inoltre, il I8 luglio, Ambrogio Lomellini scrisse al Sauli che era pronta a salpare la nave da quaranta pezzi di cannone agli ordini di Gio. Francesco Garibaldo, uno dei tanti capitani genovesi, in servizio sulle rotte atlantiche in quel momento. Le navi usate per la tratta, reperite dai diversi operatori genovesi ma anche dall'abile Francesco Feroni, una volta scaricato «l'oro nero», imbarcavano merci diverse, tra cui le cocciniglie, l'indaco e altre merci coloniali, oltre naturalmente all'argento. Uno di questi carichi venne fatto assicurare dal Sauli per la considerevole cifra di ventottomila pezzi da otto. Inoltre, nella stessa estate del I666 il clan genovese di Madrid era riuscito a firmare un asiento per il noleggio di una piccola squadra di vascelli agli ordini di Ippolito Centurione, con l'incarico di scortare dalle Canarie fino a Cadice le flotte provenienti dall'America. Insomma, il sistema si presentava articolato per organizzazione e distribuzione di funzioni tra i diversi operatori impiegati. Nel periodo a cavallo tra gli anni Sessanta e Settanta il meccanismo raggiunse la piena maturazione: le navi coprivano l'intero percorso triangolare tra l'Europa, l'Africa, l'America e il ritorno in Europa. Un solo esempio: agli inizi del 1668 Domenico Grillo chiese a Francesco Maria Sauli di assicurare il vascello Santa Cruz, comandato dal capitano Gio. Francesco Garibaldi, di 500 tonnellate, 34 cannoni e 70 uomini di equipaggio, in partenza da Cadice e diretto in Angola, ${ }^{63}$ dove avrebbe caricato schiavi. Da qui la nave avrebbe proseguito per Curaçao e, dopo aver sbarcato una parte del carico, avrebbe proseguito per Santo Domingo e Portorico, dove avrebbe terminato la consegna degli schiavi. A questo punto, una volta caricate merci varie per Cumanà e Caracas, finito lo sbarco, avrebbe imbarcato un nuovo carico di casse d'argento dirette a Cadice. ${ }^{64}$ Qui la nave Santa Cruz avrebbe ritrovato il convoglio della Repubblica, che in ultima battuta avrebbe condotto il prezioso carico a Genova. È interessante notare come in questo contesto atlantico i ge-

63. João Medina e Isabel Castro Henriques, A rota dos escravos. Angola e a rede do comércio negreiro, Cegia, Lisboa, 1996.

64. AGI, Contratación, leg. 4954. 
novesi, fin dalla metà degli anni Cinquanta, avevano cominciato a frequentare il porto di Luanda al fine di caricare schiavi e altre merci africane. Nel dicembre 1655, infatti, Agostino Castelletto, a seguito del sequestro della sua nave giunta nel porto angolano nel corso del mese di giugno di quell'anno, chiese al Consiglio Ultramarino di Lisbona l'autorizzazione per poter accedere al porto di Luanda liberamente, considerato anche che in quel momento il Portogallo si era ribellato alla Spagna e pertanto gli alleati genovesi erano considerati nemici. ${ }^{65} \mathrm{~A}$ seguito di una Consulta del Consiglio Ultramarino del 9 gennaio I656, si diede il benestare al transito nel porto di Luanda di navi genovesi di competenza di Agostino Castelletto, ma anche di Francesco Maria Rosso. Analogamente, la compagnia Grillo e Lomellini, per poter inviare le proprie navi a Luanda a caricare schiavi, chiese ed ottenne l'autorizzazione ufficiale da parte dei portoghesi il is febbraio I666. ${ }^{66}$

Gli esempi si potrebbero moltiplicare, ma quel che è importante sottolineare in questa sede è l'estensione della rete genovese che, grazie all'armamento marittimo ligure, si sviluppò attraverso l'Atlantico per poi connettersi, tramite Cadice e Siviglia, ai traffici del Levante del Mediterraneo, dove la merce più richiesta era proprio l'argento americano. ${ }^{67}$

Mentre alcuni membri del patriziato genovese erano impegnati nel traffico degli schiavi e nel commercio atlantico, altri, capeggiati dal clan Durazzo, nel I665, con un colpo di mano, avevano riaperto il commercio agevolato con l'impero ottomano e ciò aveva permesso di legare il traffico atlantico a quello levantino, favorendo così l'afflusso

65. Arquivo Histórico Utramarino (AHU), Conselho Ultramarino, Angola, caixa 6, docc. 35-42-43/r. La nave sequestrata era la Nostra Signora della Guadalupe, comandata da Giovanni Battista Piuma (9 febbraio 1656). Sul funzionamento del Consiglio Ultramarino si veda: Edval De Souza Barros, «Negócios de tanta importância» in $O$ Conselho Ultramarino e a disputa pela condução da guerra no Atlântico e no Índico (I643-I66I), CHAM, Lisboa, 2008.

66. AHU, Conselho Ultramarino, Angola, caixa 9, doc. I3, I9 febbraio I666.

67. Lo BAsso, Capitani, corsari e armatori, pp. 33-36. 
d'argento verso l'Asia. ${ }^{68}$ In cambio del metallo prezioso, gli operatori liguri furono autorizzati a commerciare merci levantine, caricabili nel porto di Smirne, dove tradizionalmente confluivano le carovane orientali. ${ }^{69}$ Ben presto però i genovesi, sulla scia, per la verità, di quel che già facevano i francesi, cominciarono a riversare in Levante argento sotto forma di luigini, una piccola moneta molto richiesta dagli ottomani. Nel giro di poco tempo gli operatori liguri, sfruttando l'affare, iniziarono a introdurre nei territori ottomani una quantità straordinaria di monete adulterate. Tra il I667 e il I669 il tasso d'argento scese notevolmente: dapprima si coniarono monete con otto parti di metallo prezioso a fronte di quattro parti di lega - e per questo erano chiamati ottavetti -, ma successivamente si produssero luigini con un tenore d'argento inferiore anche al 50\%. La frode fu così clamorosa da essere definita da Carlo Maria Cipolla come la «truffa del secolo» ed impegnò i traffici e gli affari in Levante fino a metà degli anni Settanta del secolo, quando gli ottomani cominciarono ad ostacolare l'attività dei genovesi fino a decretarne l'espulsione dei rappresentanti ufficiali nei primi anni Ottanta.

Firmate nel 1665, con un colpo di mano di Gian Agostino Durazzo, le capitolazioni con la Sublime Porta avrebbero dovuto dare nuovo vigore all'economia genovese, in difficoltà nel settore manifatturiero, in particolare in quello tessile. ${ }^{70}$ Una delle idee di fondo era di favorire la

68. Il commercio dell'argento che da Cadice giungeva a Genova per poi ripartire via Livorno e Napoli verso il Levante è accennato nella lettera del 6 febbraio 1668 scritta da Marcello Durazzo al suo agente a Napoli Andrea Terralavoro (ADGG, Archivio Durazzo, leg. I79, c. I5r).

69. Archivio Spinola di Pellicceria di Genova (ASPG), leg. 394, copialettere di Battista Durazzo, lettera a Marcello Durazzo del 3I gennaio I668: «Di giorno in giorno si attende però di Persia una ricca Caravana et essendo la seta massime in vari prezzi e senza domanda e forza, che li armeni applichino di nuovo a spedirla per loro conto di Ponente e con questo si metteranno in piedi li cambi marittimi, che per altro saranno a prezzi tenui per li molti che vi applicano, e per l'abbondanza di luigini, quali restano sempre la moneta più gradita».

70. Sui Durazzo protagonisti di questa avventura levantina, tra i tanti studi, mi limito a segnalare il contributo di Giovanni Assereto, «I Durazzo di Palazzo Reale. 
costituzione di un opificio che avrebbe dovuto produrre panni da smerciare in Levante, oltre naturalmente al traffico d'argento.

Il luigino era molto apprezzato nell'Impero Ottomano, più per il lato estetico che per la qualità della lega, e dopo il I656 esplose una vera e propria mania per questa moneta. Il suo grande successo portò molti mercanti francesi a voler aumentare ulteriormente i guadagni, stampando monete dal valore intrinseco sempre minore, contenenti meno argento e più rame di quanto stabilito dalla zecca di Sua Maestà Cristianissima. Gli speculatori si rivolsero, per il conio dei luigini alterati, a quei feudatari che possedevano l'antico diritto di battere moneta, come la principessa di Dombes e il principe di Orange. Furono quindi i francesi i primi a coniare e utilizzare queste monete di bassa lega e a smerciarle tramite lo scalo di Smirne.

La prospettiva di enormi guadagni non passò inosservata agli operatori commerciali genovesi, che colsero l'occasione e si mossero rapidamente per intraprendere la stessa strada dei francesi. Molti patrizi affittarono le proprie zecche, situate in feudi che non cadevano sotto la giurisdizione della Repubblica, a speculatori connazionali o stranieri. Si possono ricordare i Cibo con la zecca di Massa, i Grimaldi a Monaco, gli Spinola a Tassarolo e Ronco, i Doria a Loano e Torriglia, i Centurione-Scotti a Campi, i Malaspina a Fosdinovo e altri ancora. Lo scandalo scoppiò nel i667 a causa delle lamentele degli inglesi di Livorno, in quel momento tagliati fuori dagli affari levantini. Non si può escludere che anche i britannici abbiano preso parte a questa speculazione, ma certo è che, quando appurarono la bassissima qualità di molti luigini provenienti da Smirne, protestarono vivacemente presso il Sultano. Gli ottomani, a loro volta, presentarono le loro rimostranze alla Francia, accusandone il governo di favorire lo spaccio di tale moneta contraffatta. Naturalmente la risposta della corte di Parigi fu immediata, almeno dal punto di vista formale. A partire dal I670, le iniziative dei singoli governi e il rifiuto dei commercianti locali di ricevere pagamenti con

Breve storia di una grande famiglia patrizia», in L. Leoncini, ed., Da Tintoretto a Rubens. Capolavori della collezione Durazzo, Skira, Milano 2004, pp. 25-39 
quella moneta, ridimensionarono il problema. Tuttavia, bisogna segnalare il comportamento di alcuni esponenti delle più importanti famiglie genovesi, che non si fecero alcuno scrupolo a partecipare all'affare, riuscendo così a legare gli affari levantini con quelli atlantici. Tra costoro, un ruolo rilevante lo ebbero i Durazzo. ${ }^{71}$

Nel periodo 1659-1667, alcune delle principali cariche dello Stato e del corpo diplomatico erano ricoperte da membri di questa famiglia: Cesare Durazzo fu doge tra il I665 e il I667; Gian Luca Durazzo, tra il I659 e il I666, fu ambasciatore nelle principali capitali europee, tra cui Parigi, Milano e Londra; Giovanni Agostino ottenne nel 1665, durante il primo anno di dogato dello zio Cesare, le capitolazioni dal Sultano. Figura di primo piano della famiglia, anche se non ricopriva ruoli pubblici, era Marcello Durazzo. Il Marchese di Gabiano aveva accumulato ingenti ricchezze grazie alle sue attività nel settore marittimo, con una predilezione per le speculazioni assicurative, e per questo era legato ad altri membri del patriziato che negli stessi anni avevano rilanciato le attività armatoriali. Certamente fu il cervello dell'operazione, come è dimostrato dalle lettere che inviava e riceveva dai suoi agenti, collaboratori e parenti collocati nei maggiori scali commerciali. ${ }^{72}$ Marcello sfruttava questa sua rete di informatori per dare direttive, consigliare i migliori investimenti da fare, ricevere notizie riguardo lo smercio dei luigini. Fitta è la corrispondenza con Domenico Nascio, agente a Livorno, che diventò, nonostante i divieti del granduca, il porto principale per lo smercio delle monete false verso il Levante. Qui venivano caricate su bastimenti che si dirigevano verso Smirne, dove ad occuparsi dello sbarco c'erano un altro Durazzo, Battista, cugino di Marcello, e Giuseppe Nascio, fratello di Domenico.

Il sistema usato, probabilmente ideato da Marcello, era semplice: le monete false venivano coniate in zecche che non ricadevano sotto la

7I. Pastine, Genova e l'Impero Ottomano, pp. 6I-89. Sulle vicende dei Durazzo in Levante si veda il lavoro fondamentale di Fausto Fioriti, «Battista Durazzo a Smirne (I668-1669): finanza, commercio e monete false», tesi di master, Università degli Studi di Genova, a.a. 20I3-I4.

72. ADGG, Archivio Durazzo, leg. II3-I79-I80. 
giurisdizione della Repubblica, come ad esempio quella di Loano, che Eugenio Durazzo, altro cugino di Marcello, nonché fratello dei due ambasciatori, aveva preso in affitto dalla principessa Violante Lomellini Doria. Venivano quindi trasportate a Livorno o a Genova nascoste sotto le altre merci per eludere i controlli e i divieti. A questo punto entrava in gioco Marcello Durazzo con la sua esperienza di assicuratore e investitore: impegnava, o dava ordine ai suoi di farlo, i luigini nei cambi marittimi, ${ }^{73}$ cioè prestava denaro a un capitano o ad un padrone marittimo che, una volta giunto a destinazione, avrebbe dovuto ripagarlo in reali da otto spagnoli. I luigini erano poi portati all'incaricato del Durazzo che li avrebbe successivamente smerciati, usandoli per acquistare prodotti locali (pelli, sete e altro) da rispedire in patria o rinvestendoli in altri cambi marittimi.

Insomma, i genovesi attuarono nei porti turchi la stessa strategia delle potenze commerciali che da tempo vi si erano ritagliate uno spazio privilegiato: provarono ad attingere il più possibile alle risorse dell'entroterra anatolico, siriano e persiano, riversando, nel corso dei viaggi di andata, i prodotti delle proprie attività artigianali e proto-industriali (soprattutto tessuti, ma anche carta), sulla scia dei luigini.

Il pellame, invece, è il protagonista assoluto dell'import genovese dal Levante: dai registri doganali genovesi ${ }^{74}$ si ricava che di ventitré grandi navi indicate come provenienti dagli scali del Gran Signore (Smirne è prevalente, ${ }^{75}$ come detto) ben tredici risultano scaricare consistenti partite di pelli: in un caso - il I9 maggio I670 - il capitano della San Sebastiano dichiarò di aver portato con sé 4800 «pezze vaccine

73. Lo Basso, Capitani, corsari e armatori, pp. 27-32.

74. Il registro in questione è il leg. 315 (ASG, Banco di San Giorgio, sala 37).

75. David Abulafia, Il grande Mare. Storia del Mediterraneo, Mondadori, Milano, 2013, pp. 454-459. Edhem Eldem, Daniel Goffmann e Bruce Masters, The Ottoman City between East and West. Aleppo, Izmir and Istanbul, Cambridge University Press, Cambridge, 1999, pp. 79-134; Daniel Goffmann, Izmir and Levantine World (I550-I650), University of Washington, Seattle, 1990; Léon KonTEnTÉ, Smyrne et l'Occident. De l'Antiquité au XXIème siècle, Ivelinédition, Montigny le Bretonneux, 2005, pp. 285-338. 
pellose» per conto di Eugenio Durazzo, grande mercante del clan. Sempre sulla San Sebastiano - insieme alla Sant'Antonio Abate, la più avvezza ai viaggi di Levante in quegli anni - viaggiarono nell'estate seguente I82I "pezze» della stessa qualità, sbarcate a Genova il 5 luglio e consegnate al Giovanni Agostino Durazzo, l'artefice del successo del I665. Inoltre, secondo alcuni manifesti di bordo di navi di ritorno da Smirne in quegli stessi anni, sempre la Sant'Antonio Abate, capitanata da Pietro Ferrari, portò indietro nel corso del 1668 pellame, seta, lana, cotone filato e altre mercanzie proprio per conto di Eugenio Durazzo e Vincenzo Spinola; e i due erano i commissionari principali di un'altra spedizione della San Sebastiano, condotta da capitan Bastiano Tixi, che l'8 agosto I667 salpò da Smirne con le solite merci, oltre a I2 sacchi di cera. ${ }^{76}$ Il viaggio della nave di Gerolamo Costa, arrivata a Genova nel dicembre del $\mathrm{r} 668$, si delinea come una vera e propria impresa di famiglia: Battista ed Eugenio Durazzo risultano proprietari, tra le altre cose, di 6Io "pelli bufale» e di 48 balle di lana; mentre ai membri della famiglia Giovanni Agostino e Pietro risultano intestate altre partite di merce. A proposito della lana, in base all'esaminato registro del portofranco, il maggior carico giunto a Genova fu quello della nave San Domenico: I3I balle, acquistate il 3 luglio i67o dal solito Eugenio Durazzo. Dal manifesto di bordo della San Sebastiano, comandata da Pietro Locatelli, appare che nel settembre I668 di balle di lana se ne sbarcarono ben 203. Il binomio lana-pelli fu rotto soltanto da un paio di carichi di «cenere di Levante», destinati presumibilmente ad alimentare l'industria vetraria di Altare, sulle alture di Savona, e da un po' di pesce per le tavole patrizie: dalla Sant'Antonio Abate furono scaricati, nei primi tre giorni dell'aprile 1672, I3 botti di «morone salato" (7 per Vincenzo Spinola e 6 per Giovanni Agostino Durazzo) e 3 «caratelli» di caviale acquistati da un mercante fiammingo. ${ }^{77}$

76. ASG, Antica finanza, leg. II87. Prima di attraccare a Genova, la nave del capitano Tixi scaricò una parte del carico a Livorno, venduto in prevalenza a mercanti ebrei e inglesi.

77. Fra gli altri articoli che si possono trovare in terra ottomana, anche i tappeti: ne carica alcuni per conto di ricchi clienti (quali Stefano Lomellini, Carlo Pallavicini 
Ma ritorniamo di nuovo ai viaggi di andata verso il Levante. Oltre a Marcello, un altro Durazzo protagonista della vicenda fu Battista, il principale mercante dei luigini nel porto di Smirne. Figlio di Cesare (doge nel biennio I665-67) e di Giovanna Cervetto, Battista detto Baccio fu battezzato a Genova nella chiesa della Maddalena il 23 dicembre I637 e morì, sempre a Genova, il I8 ottobre I706. Il ricco archivio privato di Battista è conservato presso l'Archivio di Galleria di Palazzo Spinola a Genova e comprende cinque libri contabili e un copialettere. ${ }^{78}$ Proprio quest'ultimo assume una particolare importanza nella ricostruzione del sistema di smaltimento dei luigini alterati, perché copre il momento di massima espansione della truffa compreso tra il I667 e l'inverno del I669. In generale Battista eseguiva gli ordini provenienti dai membri della famiglia residenti a Genova, ma non mancava di operare con intraprendenza per quanto concerneva lo spaccio dei luigini: "Io non posso a bastanza lodarvi, che si mandino degli ottavi non potendo soffrire, che a Genova siate così lenti in non godere dell'occasione, e che s'impieghi i denari di Spagna, dove non ponno dare la metà di quello che frutteranno in Levante». ${ }^{79}$ In questo breve passaggio Battista Durazzo riassume in maniera efficace il legame tra il rientro dell'argento americano, frutto degli investimenti spagnoli, e il consecutivo smercio, sotto forma di luigini, nei territori ottomani.

Con la fine degli anni Settanta la situazione precipitò per l'opposizione del nuovo Gran Visir Kara Mustafa, che si dimostrò subito fiero nemico dei genovesi, i quali nel giro di poco tempo furono costretti ad abbandonare rapidamente il territorio ottomano o a rimanere coperti sotto altre «bandiere». Era la fine di un'epoca. Nella seconda metà degli anni Settanta, pressoché in contemporanea, mentre si esauriva il fruttuoso traffico di monete con il Levante, terminava anche il controllo diretto dell'asiento degli schiavi in Atlantico. Ciò non significò la fine

e Gio. Batta Doria) la Capitana, galea dello stuolo pubblico della Repubblica, partita da Smirne il 4 maggio 1667.

78. Oltre al copialettere (leg. 394), l'archivio di Battista Durazzo comprende i seguenti pezzi: leg. IO6-II2-I24-I43 e 36I.

79. ASPG, leg. 394, lettera a Pietro Durazzo del 29 febbraio I668. 
immediata della presenza di operatori liguri nei traffici a lungo raggio, ma ne determinò un nuovo mimetismo commerciale, più adatto in un mondo nel quale le potenze commerciali olandese, inglese e francese presero il sopravvento.

È fuor di dubbio che a partire dagli anni centrali del Seicento le operazioni commerciali e finanziarie dei genovesi si dipanarono su una rete di traffici globali, sostenuta da un rilancio in grande stile del settore armatoriale. Tramite la politica navalista, forse presuntuosa e sicuramente velleitaria, almeno sul breve periodo, gli operatori mercantili della Superba trovarono un nuovo slancio economico che si appoggiava sulla ormai solida diaspora mercantile distribuita tra l'America, il Mediterraneo, l'Africa e l'Asia, dove si interconnetteva con altre reti commerciali come quella armena, ebraica o musulmana, secondo la logica del commercio interculturale tanto caro a Philip Curtin e ai più recenti studi di Francesca Trivellato, di Guillaume Calafat e di Wolfgang Kaiser. ${ }^{80}$ In pochi anni, sfruttando anche antiche logiche legate al sistema imperiale spagnolo degli Austrias, i genovesi riuscirono a collegare gli scambi atlantici tra l'Europa, l'Africa e l'America, basati in larga misura sul traffico degli schiavi e su quello dell'argento, con i tradizionali scambi con il Levante Mediterraneo, vero snodo del commercio mondiale dell'epoca. Tra i primi anni Sessanta e la metà del decennio successivo, alcune compagnie socialmente miste (questa è una delle tante novità), composte di nobili e di ricchi esponenti della cosiddetta borghesia mercantile, riuscirono ad interconnettere i porti americani - Curaçao, Caracas, Vera Cruz, Cartagena, Portobelo - con quelli europei - Lisbona, Cadice-Siviglia, Genova e Smirne -, con il terminale africano di Luanda in Angola e con quello asiatico di Manila, muovendo uomini e merci in tutte le direzioni, lucrando su ogni aspetto legato al mondo dello shipping - prestiti marittimi, noli, assicurazioni - e controllando le rotte sul piano strettamente navale, in un gioco che si

80. Wolfgang Kaiser e Guillaume Calafat, «The Economy of Ransoming in the Early Modern Mediterranean. A Form of Cross-Cultural Trade between Southern Europe and the Maghreb (Sixteenth to Eighteenth Centuries)», in Religion and Trade, pp. I08-I30. 
articolava come da tradizione su una miscela di iniziative private e di scelte strategiche operate all'interno degli organi repubblicani. Nel giro di pochi anni fu proprio il potenziamento della politica marittima dello Stato genovese, incardinato sulla creazione di nuove e moderne istituzioni, a dare luogo a questa piccola globalizzazione «alla genovese», all'avanguardia per tecniche mercantili e nautiche. Insomma, lungi da essere quello Stato-non Stato, dalle deboli capacità istituzionali, la Repubblica di Genova fu, tra Seicento e Settecento, un esempio di modernità in linea con le grandi potenze europee. 


\title{
Memòria final del VIIIè Congrés d'Història Moderna de Catalunya
}

\author{
Mariela Fargas Peñarrocha \\ Universitat de Barcelona \\ Secretària acadèmica del VIIIè Congrés \\ d'Història Moderna de Catalunya
}

Sr. director del VIIIè Congrés d'Història Moderna de Catalunya, senyores i senyors, congressistes, professorat de l'Àrea d'Història Moderna de la Facultat de Geografia i Història de la Universitat de Barcelona — de fet, impulsors del Congrés—, estudiants: el VIIIè Congrés d'Història Moderna de Catalunya, "Catalunya i el Mediterrani», ha arribat a la seva cloenda. Comença el temps, fructífer, de recapitulació i valoració dels resultats, dels treballs i de la recerca presentada, plantejada, debatuda, que adquirirà la seva forma definitiva quan assistim a la publicació de les actes. Com és ja un costum arrelat, les conferències i ponències del VIIIè Congrés seran publicades íntegrament en format paper dins la revista de l'Àrea, Pedralbes. Revista d'Història Moderna. Les comunicacions també seran publicades en el marc de la revista, però en format digital. Tot el conjunt, a la vista de la rica dinàmica del Congrés, constituirà una aportació ben significativa a la historiografia general de l'època moderna i a la historiografia catalana en concret.

Però ara, amb la presentació d'aquesta memòria, és moment de fer balanç d'algunes de les dades fonamentals que han caracteritzat el Congrés.

En primer lloc, farem una breu referència a la temàtica i al seu ressò, "Catalunya i el Mediterrani», que és el resultat de les investigacions 
dutes a terme en el marc de l'Àrea d'Història Moderna i del Grup d'Estudis d'Història del Mediterrani Occidental, amb categoria de grup de recerca consolidat per l'Agència per a la Qualitat del Sistema Universitari de Catalunya en la seva darrera convocatòria. També es tracta d'un tema coherent amb la docència del professorat de l'Àrea emmarcada dins el Màster en Història i Identitats en el Mediterrani Occidental, que compartim amb les universitats de València, Jaume I i d'Alacant. Finalment, cal no oblidar que el Mediterrani com a problema històric esdevé més que mai una qüestió cabdal dins la preocupant realitat que està protagonitzant en l'actualitat. Aquesta perspectiva, en definitiva, ha rebut una importantíssima resposta, ja que han estat cent vuit els inscrits i setanta les comunicacions que s'han presentat. A aquestes comunicacions, cal sumar-hi ponències i conferències, que mostren la rellevància i l'actualitat del tema. En efecte i com ja és habitual, el Congrés s'inicia i finalitza amb dues conferències impartides per investigadors d'àmbit internacional: Brigitte Marin, de la Universitat d'Aix-Marseille, i Luca Lo Basso, de la Università degli Studi di Genova. Parallelament, el curs del Congrés s'ha estructurat en sis sessions, cadascuna, alhora, encapçalada per una ponència impartida per reconeguts professors de les universitats Autònoma de Barcelona, de Girona, Rovira i Virgili, de València, d'Alacant i de les Illes Balears, amb la idea que aquest Congrés consolidi les relacions de recerca ja establertes amb altres collegues modernistes de centres del Mediterrani occidental. Recordem els títols d'aquestes sessions: la primera, «Poders, fronteres i identitats»; la segona, «Paisatge històric i risc natural»; la tercera, «Món urbà i món rural»; la quarta, «Família, xarxes i societat»; la cinquena, «Transferències i circulacions», i la sisena i última, «Creences i vivències».

A través de les seccions indicades hem vist desfilar conflictes armats, expedicions militars valorades de nou, escenaris de negociacions postbèllliques, perspectives transversals dels conflictes, conflictes al mar, fronteres religioses, usos del bosc, altres usos dels espais per les comunitats, demografia, treball i món gremial, xarxes de comerç, moviments de població, viatges, grups socials, elits i família, mecenatge, cultura i comunicació... Una gran varietat de temes enclavats en l'espai 
que es dibuixaria entre Barcelona, Girona, Lleida, Tarragona, Andorra, València, Mallorca, Milà, Nàpols i Sicília, Sardenya, Roma i Malta, prova de la mediterraneïtat de la recerca presentada.

Si les aportacions han estat diverses, també ho són les procedències dels participants. Els seus autors són investigadors de diferents universitats, catalanes, de València, de les Illes Balears, d'universitats espanyoles i estrangeres. Aquesta és part de la riquesa del Congrés, de la seva molt positiva acollida, de la seva àmplia participació.

Però la participació avui dia té una doble dimensió: la real i la virtual. Tal com ja es va fer en l'edició anterior, la participació s'ha multiplicat gràcies a la informació vessada permanentment al Twitter de l'Àrea, que gairebé s'està acostant als dos mil seguidors i on s'han retransmès en directe totes les conferències i ponències, que han estat visualitzades en el seu conjunt per quasi dos-cents espectadors. Podem dir que el VIIIè Congrés ha arribat a molts llocs i molts moments. Paral.lelament, en aquesta edició hem obert un compte a Instagram amb la mateixa finalitat: fer arribar a un públic més jove el coneixement forjat en la nostra trobada científica.

Si parlem de participació, cal al.ludir a les colllaboracions, i en aquest punt hem de fer dues mencions especials: primerament, del concert d'orgue que ha tingut lloc dins la segona jornada del Congrés a l'església dels Sants Just i Pastor de Barcelona, a càrrec de l'organista Jordi Pajares Ribas, que ens va oferir un programa amb peces de compositors del segle Xvi al xx, d'Antonio de Cabezón, Johann Sebastian Bach, Amadeus Mozart, Josef Rheinberger, Olivier Messiaen; en segon lloc i dins la tercera jornada del Congrés, cal esmentar la visita a Tarragona, que s'inscriu dins la llarga tradició de relacions culturals que els diferents congressos d'Història Moderna de Catalunya han conreat fora de Barcelona com a expressió de la voluntat d'estar-hi present i d'apropar-se a una diversitat d'entorns. Aquesta visita, amb una programació variada que ha mostrat l'arxiu de Tarragona a l'antic convent de Sant Francesc, l'església de Sant Agustí, l'antic convent de Sant Domènec, la casa Castellarnau i l'edifici de l'antiga Audiència, ha estat dinamitzada pel professor Josep Fàbregas, de la Universitat Rovira i Virgili, a qui també agraïm la seva disponibilitat. 
Parlant d'agraïments, n'hem d'adreçar a totes les persones i institucions que amb el seu ajut han fet possible la realització del VIIIè Congrés. Al Departament de Disseny i Imatge de la Facultat de Belles Arts de la Universitat de Barcelona, que, un cop més, com en altres edicions, ha organitzat un concurs per dissenyar la imatge gràfica del Congrés, al professor Enric Tormo, impulsor d'aquest concurs, a tots els alumnes concursants i, especialment, a la guanyadora, Sandra Bossoms, a qui felicitem particularment. També molt especialment a l'equip de voluntaris, quinze alumnes de la Facultat, del nostre grau d'Història, que en l'actualitat estan cursant assignatures ofertes per l'Àrea d'Història Moderna i que amb la seva illusionada participació han assegurat els aspectes més pràctics i de logística. Aquest grup de voluntaris ha estat dirigit pels professors Ida Mauro i Diego Sola, que han tingut un paper molt rellevant en els treballs del VIIIè Congrés. I hem de fer arribar tot el nostre reconeixement a les institucions: a la Facultat de Geografia i Història, al Vicerectorat de Recerca, a la Universitat Rovira i Virgili, a la Diputació de Barcelona, a la Fundació Noguera, al Museu Marítim de Barcelona, a la Fundació Mútua Catalana, a l'Ateneu Universitari Sant Pacià, a la Reial Acadèmia de Bones Lletres de Barcelona. Gràcies a tots ells i a les persones que els representen, quasi trenta-cinc anys després d'aquell primer Congrés del I984 -on, per cert, exercí de secretari el professor Joan Bada, que ens deixava no fa gaire-, el Congrés s'ha celebrat, com també podrà tenir lloc la publicació de les seves actes. És un esdeveniment acadèmic i de recerca molt important per a l'Àrea d'Història Moderna de la Universitat de Barcelona. Donem l'enhorabona, per tant, al professor Jaume Dantí, coordinador de la Secció d'Història Medieval i Moderna, on s' insereix la nostra Àrea, per la seva importantíssima execució. I, confiats en la positiva resposta rebuda, tanquem el VIIIè Congrés amb la mirada posada en el proper, el IXè. 